\title{
A New Analytical Approach for Solving Nonlinear Boundary Value Problems in Finite Domains
}

\author{
Jafar Biazar ${ }^{1}$, Behzad Ghanbari ${ }^{2 *}$ \\ ${ }^{1}$ Department of Applied Mathematics, Faculty of Mathematical Sciences, University of Guilan, Rasht, Iran \\ ${ }^{2}$ Department of Mathematics, Kermanshah University of Technology, Kermanshah, Iran \\ E-mail: b.ghanbary@yahoo.com \\ Received November 4, 2010; revised May 25, 2011; accepted July 4, 2011
}

\begin{abstract}
Based on the homotopy analysis method (HAM), we propose an analytical approach for solving the following type of nonlinear boundary value problems in finite domain. In framework of HAM a convenient way to adjust and control the convergence region and rate of convergence of the obtained series solutions, by defining the so-called control parameter $\hbar$, is provided. This paper aims to propose an efficient way of finding the proper values of $\hbar$. Such values of parameter can be determined at the any order of approximations of HAM series solutions by solving of a nonlinear polynomial equation. Some examples of nonlinear initial value problems in finite domain are used to illustrate the validity of the proposed approach. Numerical results confirm that obtained series solutions agree very well with the exact solutions.
\end{abstract}

Keywords: Homotopy Analysis Method, Boundary Value Problems, Finite Domain

\section{Introduction}

In this work, we consider the following type of nonlinear boundary value problems in a finite domain (as considered in [1]):

$$
u^{(n)}(x)=f\left(x, u, u^{\prime}, \cdots, u^{(n-1)}\right), \quad a \leq x \leq b
$$

subject to the two-point boundary conditions

$$
\begin{aligned}
& u(a)=\alpha_{0}, u^{\prime}(a)=\alpha_{1}, \cdots, \quad u^{(r)}(a)=\alpha_{r} \\
& u(b)=\beta_{0}, u^{\prime}(b)=\beta_{1}, \cdots, \quad u^{(n-r-2)}(b)=\beta_{n-r-2}
\end{aligned}
$$

where $0 \leq r \leq n-2$ is an integer.

Also $f$ is a polynomial in and

$x, u(x) u^{\prime}(x), \cdots, u^{(n-1)}(x), \quad \alpha_{0}, \alpha_{1}, \cdots, \alpha_{r}, \beta_{0}, \beta_{1}, \cdots, \beta_{n-r-2}$ are real constants.

Since, such type of boundary value problems arise in the mathematical modeling of the viscoelastic flows and other branches of mathematical, physical and engineering sciences, the approximate solutions of these problems are of great importance. See [2-4] and the references therein.

Some numerical and analytical methods such as shooting method [5], Finite-element method [6], sincGalerkin method [7], finite-difference method [8], Adomian technique [9], variational iteration method (VIM)
[10], homotopy perturbation method (HPM) [11], analysis method (HAM) [12], have been studied for obtaining approximate solutions to boundary value problems.

The homotopy analysis method [13-17] is a general analytic approach to get series solutions of various types of nonlinear equations, including ordinary differential equations, partial differential equations, differential-integral equations, differential-difference equation, and coupled equations of them. Unlike perturbation methods, the HAM is independent of small/large physical parameters, and thus is valid no matter whether a nonlinear problem contains small/large physical parameters or not. More importantly, different from all perturbation and traditional non-perturbation methods, the HAM provides us a simple way to ensure the convergence of solution series, and therefore, the HAM is valid even for strongly nonlinear problems. Besides, different from all perturbation and previous non-perturbation methods, the HAM provides us with great freedom to choose proper base functions to approximate a nonlinear problem.

These advantages make the method to be a powerful and flexible tool in mathematics and engineering, which can be readily distinguished from existing numerically and analytically methods.

Up to now, this method has been successfully applying this method to various nonlinear problems in science and 
engineering. A systematic description of the method and its applications are found in [15].

This paper is arranged in the following manner. In Section 2, the HAM is applied to solve the problem of nonlinear boundary value problems. In Section 3, the basic idea of the present approach is described. Furthermore, some numerical examples are presented in Section 4. Finally, conclusions are drawn in Section 5.

\section{The Implement of HAM to BVPs}

In order to obtain a convergent series solution to the nonlinear problem $(1,2)$, we first construct the zeroth order deformation equation

$$
(1-p) L\left[\phi(x ; p)-u_{0}(x)\right]=p \hbar N[\phi(x ; p)]
$$

where $p \in[0,1]$ is an embedding parameter, $\hbar \neq 0$ is a convergence-control parameter, and $\phi(x ; p)$ is an unknown function, respectively. According to (1), the auxiliary linear operator $L$ is given by

$$
L[\phi(x ; p)]=\frac{\partial^{n} \phi(x ; p)}{\partial x^{n}}
$$

and the nonlinear operator $N$ is given by

$$
N[\phi(x ; p)]=\frac{\partial^{n} \phi}{\partial x^{n}}-f\left(x, \phi, \frac{\partial \phi}{\partial x}, \cdots, \frac{\partial^{n-1} \phi}{\partial x^{n-1}}\right)
$$

From (3), when $p=0$ and $p=1$,

$$
\phi(x ; 0)=u_{0}(x)
$$

and

$$
\phi(x ; 1)=u(x)
$$

both hold. Therefore, as $p$ increases from 0 to 1 , the solution $\phi(x ; p)$ varies from the initial guess $u_{0}(x)$ to the solution $u(x)$. Expanding $\phi(x ; p)$ in Taylor series with respect to $p$, one has

$$
\phi(x ; p)=\phi(x ; 0)+\sum_{i=1}^{\infty} u_{i}(x) p^{i}
$$

where

$$
u_{m}(x)=\left.\frac{1}{m !} \frac{\partial^{m} \phi(x ; p)}{\partial p^{m}}\right|_{p=0}
$$

Assuming that the series (6) is convergent at $p=1$, The solution series

$$
u(x)=\phi(x ; 1)=u_{0}(x)+\sum_{i=1}^{\infty} u_{i}(x)
$$

must be one of the solutions of the original problem (1, $2)$, as proved by Liao in [15].

Our next goal is to determine the higher order terms $u_{m}(x)(m \geq 1)$. Define the vector

$$
\boldsymbol{u}_{s}(x)=\left\{u_{0}(x), u_{1}(x), \cdots, u_{s}(x)\right\}
$$

Differentiating the zeroth order deformation Equation (3) $m$ times with respect to $p$, then setting $p=0$, finally dividing them by $m$ !, we obtain the $m$ th order deformation equation

$$
L\left[u_{m}(x)-\chi_{m} u_{m-1}(x)\right]=h R_{m-1}(x)
$$

and its boundary conditions

$$
\begin{gathered}
u_{m}(a)=u_{m}^{\prime}(a)=\cdots=u_{m}^{(r)}(a)=0 \\
u_{m}(b)=u_{m}^{\prime}(b)=\cdots=u_{m}^{(n-r-2)}(b)=0
\end{gathered}
$$

where

$$
R_{m-1}(x)=\left.\frac{1}{(m-1) !} \frac{\partial^{m-1} N[\phi(x ; p)]}{\partial p^{m-1}}\right|_{p=0}
$$

and

$$
\chi_{m}= \begin{cases}1, & m>1 \\ 0, & m \leq 1\end{cases}
$$

From (4), $m$ th order deformation Equation (10) becomes

$$
u_{m}^{(n)}(x)=\chi_{m} u_{m-1}^{(n)}(x)+h R_{m-1}(x)
$$

In this way, the component solutions of $u_{m}, m \geq 1$, are not only dependent upon $x$ but also the auxiliary parameter $\hbar$. Thus, the convergence of the series (8) depends on the parameter $\hbar$.

Finally, the $m$ th order approximation to the problem $(1,2)$ can be generally expressed by

$$
\tilde{u}_{m}(x)=u_{0}(x)+\sum_{i=1}^{m} u_{i}(x)
$$

As we know, to find a proper convergence-control parameter $\hbar$, to get a convergent series solution or to get a faster convergent one, there is a classic way of plotting the so-called " $\hbar$-curves" or "curves for convergencecontrol parameter”. For example, one can consider the convergence of $u^{\prime}(x)$ and $u^{\prime \prime}(x)$ of a nonlinear differential equation $N[u(x)]=0$ to find a region say $R_{\hbar}$ so that, each $\hbar \in R_{\hbar}$ gives a convergent series solution of such kind of quantities.

Such a region can be found, although approximately, by plotting the curves of these unknown quantities versus $\hbar$.

However, it is a pity that curves for convergence-control parameter (i.e. $\hbar$-curves) give us only a graphically region and cannot tell us which value of $\hbar \in R_{\hbar}$ gives the fastest convergent series. Furthermore, recently in 
[18] a misinterpreted usage of $\hbar$-curves has reported.

Although the solution series (15) given by different values in the valid region of $\hbar$ converge to the exact solution, the convergence rates of these solution series are usually different.

\section{Proposed Approach}

It should be noted that based on the zeroth-order deformation Equation (3), $m$ th-order HAM approximation of the solution $u(x)$, giving by

$$
\tilde{u}_{m}(x)=u_{0}(x)+\sum_{i=1}^{m} u_{i}(x)
$$

is also dependent upon the convergence-control parameter $\hbar$.

For $\theta=\frac{1}{2}(b+a)$, let

$$
\begin{aligned}
P_{m}(\hbar) & =N\left[\tilde{u}_{m}(\theta)\right] \\
& =\tilde{u}_{m}^{(n)}(\theta)-f\left(\theta, u(\theta), u^{\prime}(\theta), \cdots, u^{(n-1)}(\theta)\right) \\
& =0
\end{aligned}
$$

donate the residual error of the governing Equation (12) at the $m$ th-order of HAM approximation.

Since $P_{m}(\hbar)=0$ is indeed a polynomial equation of order $m$, so at each order of approximation $m$, we can gain the value of $\hbar$ by solving only one algebraic equation.

\section{Numerical Examples}

To demonstrate the efficiency of the proposed approach, we consider several examples. For comparison purpose they were taken from [1,12].

Example 1. Let's consider the following second order boundary value problem [1].

$$
u^{\prime \prime}(x)=2 u(x)^{3}-6 u(x)+2 x^{3}, 1 \leq x \leq 2
$$

with the boundary conditions

$$
u(1)=2, u(2)=\frac{5}{2}
$$

which has the exact solution in the form of

$$
u(x)=x+\frac{1}{x}
$$

For the zeroth order deformation Equation (3), the auxiliary linear operator $L$ is given by

$$
L[\phi(x ; p)]=\frac{\partial^{2} \phi(x ; p)}{\partial x^{2}}
$$

and the nonlinear operator $N$ is given by

$$
N[\phi(x ; p)]=\frac{\partial^{2} \phi(x ; p)}{\partial x^{2}}-2 \phi(x ; p)^{2}+6 \phi(x ; p)-2 x^{3}
$$

In view of the boundary conditions (18), the initial guess is determined as

$$
u_{0}(x)=x^{2}-\frac{5}{2} x+\frac{7}{2}
$$

To obtain higher order terms $u_{m}(x)$, the $m$ th order deformation Equation (10) and its boundary conditions (11) are calculated:

$$
\begin{gathered}
u_{m}^{\prime \prime}(x)=\chi_{m} u_{m-1}^{\prime \prime}(x)+h R_{m-1}(x) \\
u_{m}(1)=0, u_{m}(2)=0
\end{gathered}
$$

where

$$
\begin{aligned}
R_{m}\left(\boldsymbol{u}_{m}(x)\right) & =u_{m-1}^{\prime \prime}(x)+6 u_{m-1}(x)+2\left(1-\chi_{m}\right) x^{3} \\
& -2 \sum_{j=0}^{m-1}\left(u_{m-1-j}(x) \sum_{i=0}^{j} u_{i}(x) u_{j-i}(x)\right)
\end{aligned}
$$

In this way, we can calculate $u_{m}(x)(m \geq 1)$ recursively.

In this example, from Equation (16) with $m=10$, the proper value for $\hbar$.obtained as $\hbar=-0.364$.

As it can be seen in Table 1 and Figure 1, obtained approximate series solution agrees very well with the exact solution (19).

Example 2. As the example, let's have the following third order boundary value problem [1].

$$
u^{(3)}(t)+u(t) u^{\prime \prime}(t)-u^{\prime 2}(t)+1=0,0 \leq x \leq 1
$$

with the boundary conditions

$$
u(0)=u^{\prime}(0)=u(1)=0
$$

This problem was considered in [9] via the finite difference method.

For the zeroth order deformation Equation (3), the auxiliary linear operator $L$ is given by

$$
L[\phi(x ; p)]=\frac{\partial^{3} \phi(x ; p)}{\partial x^{3}}
$$

and the nonlinear operator $N$ is given by

Table 1. Absolute errors of different methods in Example 1.

\begin{tabular}{ccccc}
\hline$x$ & $\begin{array}{c}\text { Exact } \\
\text { solution }\end{array}$ & $\begin{array}{c}\text { Shooting } \\
\text { method [5] }\end{array}$ & $\begin{array}{c}10^{\text {th }} \text { HAM } \\
\text { for } \hbar=-0.167[1]\end{array}$ & $\begin{array}{c}10^{\text {th }} \text { HAM } \\
\text { for } \hbar=-0.364\end{array}$ \\
\hline$x=1.1$ & 2.0090909 & 0.0006723 & 0.0001009 & $4.32 \mathrm{e}-6$ \\
$x=1.3$ & 2.0692308 & 0.0021682 & 0.0001164 & $2.59 \mathrm{e}-7$ \\
$x=1.5$ & 2.1666667 & 0.0033009 & 0.0004174 & $7.44 \mathrm{e}-6$ \\
$x=1.7$ & 2.2882353 & 0.0036213 & 0.0000210 & $8.15 \mathrm{e}-6$ \\
$x=1.9$ & 2.4263158 & 0.0021849 & 0.0007363 & $1.80 \mathrm{e}-5$ \\
\hline
\end{tabular}




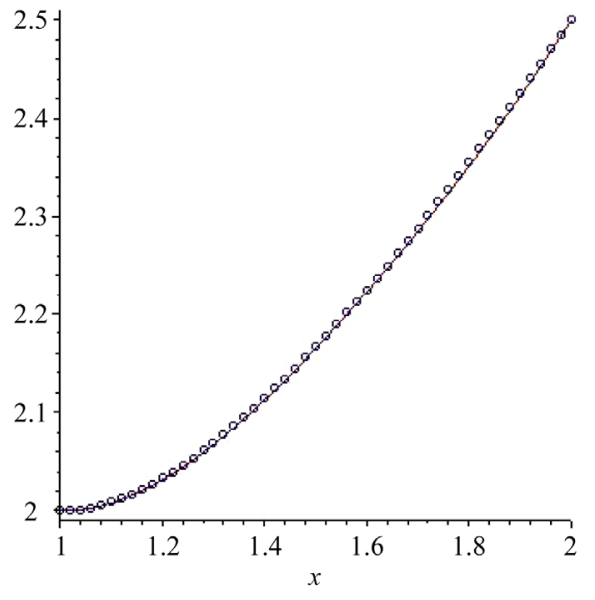

Figure 1. Symbols: 5th-order HAM approximation for $\hbar=-0.364$; solid line: exact solution.

$$
\begin{aligned}
N[\phi(x ; p)] & =\frac{\partial^{3} \phi(x ; p)}{\partial x^{3}}+\phi(x ; p) \frac{\partial^{2} \phi(x ; p)}{\partial x^{2}} \\
& =-\left(\frac{\partial \phi(x ; p)}{\partial x}\right)^{2}+1
\end{aligned}
$$

In view of the boundary conditions (21), the initial guess is determined as $u_{0}(x)=x^{3}-x^{2}$.

To obtain higher order terms $u_{m}(x)$, the $m$ th order deformation Equation (10) and its boundary conditions (11) are calculated:

$$
\begin{aligned}
& u_{m}^{(3)}(x)=\chi_{m} u_{m}^{(3)}(x)+h R_{m-1}(x) \\
& u_{m}(0)=0, \quad u_{m}^{\prime}(0)=0, \quad u_{m}(1)=0
\end{aligned}
$$

where

$$
\begin{aligned}
R_{m}\left(\boldsymbol{u}_{m}(x)\right) & =u_{m-1}^{(3)}(x)+\sum_{i=0}^{m-1} u_{i}(x) u_{m-1-i}^{\prime \prime}(x) \\
& -\sum_{j=0}^{m-1} u_{j}^{\prime}(x) u_{m-1-j}^{\prime}(x)+1-\chi_{m}
\end{aligned}
$$

In this way $u_{m}(x)(m \geq 1)$, can be done recursively.

In this case, $\hbar=-0.922$ is obtained by our approach. As shown in Table 2 and Figure 2, approximate series solution using such value of parameter, is in excellent agreement with the numerical solution given by the Runge-Kutta-Fehlberg 4-5 technique.

Table 2. Absolute errors of different methods in Example 2.

\begin{tabular}{ccccc}
\hline$x$ & $\begin{array}{c}\text { Exact } \\
\text { solution }\end{array}$ & $\begin{array}{c}\text { Shooting } \\
\text { method [5] }\end{array}$ & $\begin{array}{c}10^{\text {th }} \text { HAM for } \\
\hbar=-1.23[1]\end{array}$ & $\begin{array}{c}10^{\text {th }} \text { HAM for } \\
\hbar=-0.922\end{array}$ \\
\hline$x=0.1$ & 2.0090909 & 0.0006723 & $1.46 \mathrm{e}-6$ & $3.96 \mathrm{e}-7$ \\
$x=0.3$ & 2.0692308 & 0.0021682 & $1.41 \mathrm{e}-6$ & $3.00 \mathrm{e}-11$ \\
$x=0.5$ & 2.1666667 & 0.0033009 & $1.74 \mathrm{e}-6$ & $1.19 \mathrm{e}-8$ \\
$x=0.7$ & 2.2882353 & 0.0036213 & $2.00 \mathrm{e}-6$ & $6.89 \mathrm{e}-8$ \\
$x=0.9$ & 2.4263158 & 0.0021849 & $2.31 \mathrm{e}-6$ & $1.02 \mathrm{e}-7$ \\
\hline
\end{tabular}

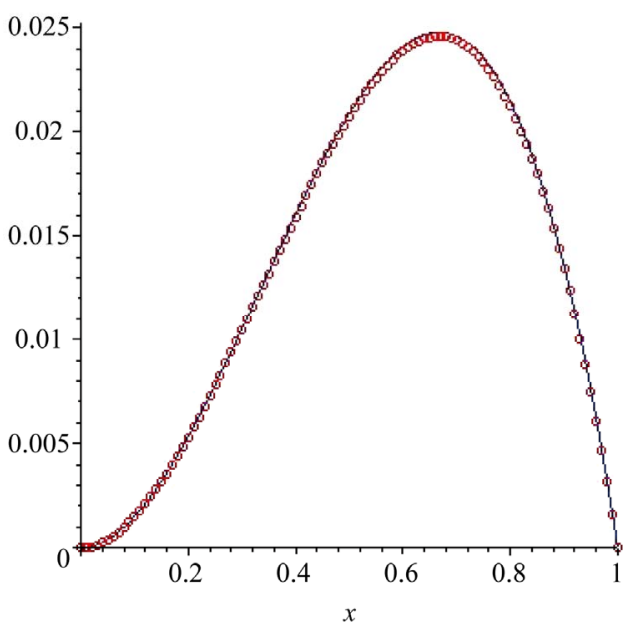

Figure 2. Solid line: 5th-order HAM approximation for $\hbar=-\mathbf{0 . 3 2 6 8 3 7 2 2}$; symbols: numerical solution.

Example 3. As the example, let's have the following nonlinear fourth-order boundary value problem involving a parameter $c$ [12].

$$
u^{(4)}(x)=c u(x)^{2}+1,0 \leq x \leq 2
$$

with the boundary conditions

$$
u(0)=u^{\prime}(0)=u(2)=u^{\prime}(2)=0
$$

For the zeroth order deformation Equation (3), the auxiliary linear operator $L$ is given by

$$
L[\phi(x ; p)]=\frac{\partial^{4} \phi(x ; p)}{\partial x^{4}}
$$

and the nonlinear operator $N$ is given by

$$
N[\phi(x ; p)]=\frac{\partial^{4} \phi(x ; p)}{\partial x^{4}}-c \phi(x ; p)^{2}-1
$$

In view of the boundary conditions (23), the initial guess is determined as

$$
u_{0}(x)=x^{4}-4 x^{3}+4 x^{2}
$$

To obtain higher order terms $u_{m}(x)$, the $m$ th order deformation Equation (10) and its boundary conditions (11) are calculated:

$$
\begin{aligned}
& u_{m}^{(4)}(x)=\chi_{m} u_{m}^{(4)}(x)+h R_{m-1}(x) \\
& u_{m}(0)=0, \quad u_{m}^{\prime}(0)=0, \quad u_{m}(2)=0, \quad u_{m}^{\prime}(2)=0
\end{aligned}
$$

where

$$
R_{m}\left(\boldsymbol{u}_{m}(x)\right)=u_{m-1}^{(4)}(x)-c \sum_{i=0}^{m-1} u_{m-1-i}(x) u_{i}(x)+\chi_{m}-1
$$

In this way $u_{m}(x)(m \geq 1)$, can be done recursively. 
Table 3. Comparisons of $\delta(t)$ of $10^{\text {th }}$-order HAM solutions for different values of $\hbar$ in Example 3.

\begin{tabular}{|c|c|c|c|c|c|c|}
\hline & $\hbar$ & $x=0.2$ & $x=0.6$ & $x=1.0$ & $x=1.4$ & $x=1.8$ \\
\hline \multirow{2}{*}{$c=1$} & $\hbar=-1 \quad[12]$ & $1.5 \mathrm{e}-2$ & & 6. $4 \mathrm{e}-3$ & $7.49 \mathrm{e}-3$ & \\
\hline & & 7.1e-7 & $4.3 e-7$ & $3.7 \mathrm{e}-7$ & & $9.6 \mathrm{e}-6$ \\
\hline \multirow{2}{*}{$c=5$} & $\hbar=-0.57 \quad[12]$ & $1.1 \mathrm{e}-2$ & $1.2 \mathrm{e}-2$ & $1.2 \mathrm{e}-2$ & $1.2 \mathrm{e}-2$ & $1.1 \mathrm{e}-2$ \\
\hline & $\hbar=-0.954$ & $1.3 e-4$ & $1.3 e-4$ & $1.2 \mathrm{e}-4$ & $1.2 \mathrm{e}-4$ & $1.4 \mathrm{e}-4$ \\
\hline \multirow{2}{*}{$c=-12$} & $\hbar=-0.634 \quad[12]$ & $1.3 e-1$ & $1.5 e-1$ & $1.5 e-1$ & $1.5 e-1$ & $1.3 e-1$ \\
\hline & $\hbar=-0.655$ & $1.0 \mathrm{e}-2$ & $9.9 \mathrm{e}-3$ & $9.4 \mathrm{e}-3$ & $9.9 e-3$ & $1.1 \mathrm{e}-2$ \\
\hline
\end{tabular}

Here we will discuss following three cases of $c$.

1) In the first case, we take $c=1$ as an example. For $c=1$ and $m=10$ in (16), we get into a nonlinear equation, which root is $\hbar=-0.924$.

2) In second case, we take $c=5$ as an example. Equation (16) with $c=5$ and $m=10$ has the real solution $\hbar=-0.954$.

3) Finally, we take $c=-12$ as an example. Equation (16) with $c=-12$ and $m=10$, introduces $\hbar=-0.655$ as a proper value for $\hbar$.

Since the closed-form solution to the problem (22), (23) is not available, the numerical solution $u_{\text {numer }}$ is calculated via the Runge-Kutta-Fehlberg 4-5 technique, then, we compare the relative errors of the 10th order HAM approximations $u_{a p p r}$ at different points in the interval $(0,1)$, using the formula

$$
\delta(t)=\left|\frac{u_{\text {appr }}-u_{\text {numer }}}{u_{\text {numer }}}\right|
$$

for different cases of $c$, are reported in Table 3.

\section{Conclusions}

In this paper the solutions of a new way of finding the control parameter in the homotopy analysis method is proposed. It is shown for obtained values of such parameter, HAM approximation series leads to exact solution of problems or produces an approximate results which are in a highly agreement with exact solution of problems. All computations were done using Maple 13 with 15 digit floating point arithmetics (Digits: $=15$ ).

\section{References}

[1] S. Liang and D. J. Jeffrey, “An Analytical Approach for Solving Nonlinear Boundary Value Problems in Finite Domains,” Numerical Algorthms, Vol. 56, No. 1, 2010, pp. 93-106.

[2] H. N. Caglar, S. H. Caglar and E. E. Twizell, "The Numerical Solution of Fifth Order Boundary-Value Problems with Sixth Degree B-Spline Functions," Applied Mathematics Letters, Vol. 12, No. 5, 1999, pp. 25-30.

\section{doi:10.1016/S0893-9659(99)00052-X}

[3] A. R. Davies, A. Karageoghis and T. N. Phillips, "Spectral Glarkien Methods for the Primary Two-Point Boundary-Value Problems in Modeling Viscelastic Flows,” International Journal for Numerical Methods in Engineering, Vol. 26, No. 3, 1988, pp. 647-662. doi:10.1002/nme.1620260309

[4] D. J. Fyfe, "Linear Dependence Relations Connecting Equal Interval Nth Degree Splines and Their Derivatives," Journal of the Institute of Mathematics and Its Applications, Vol. 7, No. 3, 1971, pp. 398-406. doi:10.1093/imamat/7.3.398

[5] S. N. Ha, “A Nonlinear Shooting Method for Two-Point Boundary Value Problems," Computers \& Mathematics with Applications, Vol. 42, No. ER10-11, 2001, pp. 14111420. doi:10.1016/S0898-1221(01)00250-4

[6] A. G. Deacon and S. Osher. "Finite-Element Method for a Boundary-Value Problem of Mixed Type," SIAM Journal on Numerical Analysis, Vol. 16, No. 5, 1979, pp. 756-778. doi:10.1137/0716056

[7] M. El-Gamel, S. H. Behiry and H. Hashish, "Numerical Method for the Solution of Special Nonlinear FourthOrder Boundary Value Problems,” Applied Mathematics and Computation, Vol. 145, No. 2-3, 2003, pp. 717-734. doi:10.1016/S0096-3003(03)00269-8

[8] A. A. Salama and A. A. Mansour, "Fourth-Order FiniteDifference Method for third-Orderboundary-Value Problems," Numerical Heat Transfer, Part B, Vol. 47, No. 4, 2005, pp. 383-401. doi:10.1080/10407790590907903

[9] A. M. Wazwaz, "The Numerical Solution of Fifth-Order Boundary-Value Problems by Adomian Decomposition”, Journal of Computational and Applied Mathematics, Vol. 136, No. 1-2, 2001, pp. 259-270. doi:10.1016/S0377-0427(00)00618-X

[10] M. Aslam Noor and S. T. Mohyud-Din, "Variational Iteration Technique for Solving Fifth-Order Boundary Value Problems,” 2006 (Preprint).

[11] C. Chun and R. Sakthivel, "Homotopy Perturbation Technique for Solving Two-Point Boundary Value Problems -Comparison with Other Methods," Computer Physics Communications, Vol. 181, 2010, pp. 1021-1024. doi:10.1016/j.cpc.2010.02.007

[12] S. Liang and D. J. Jeffrey, “An Efficient Analytical Approach for Solving Fourth Order Boundary Value Problems," Computer Physics Communications, Vol. 180, 2009, pp. 2034-2040. doi:10.1016/j.cpc.2009.06.006

[13] S. J. Liao, "On the Proposed Homotopy Analysis Technique for Nonlinear Problems and Its Applications,” Ph. D. Dissertation, Shanghai Jiao Tong University, Shanghai, 1992.

[14] S. J. Liao, “General Boundary Element Method for Nonlinear Heat Transfer Problems Governed by Hyperbolic Heat Conduction Equation,” Computational Mechanics, Vol. 20, No. 5, 1997, pp. 397-406. doi:10.1007/s004660050260

[15] S. J. Liao, "Beyond Perturbation: Introduction to Homotopy Analysis Method,” Chapman \& Hall/CRC Press, 
Boca Raton, 2003. doi:10.1201/9780203491164

[16] S. J. Liao, "Numerically Solving Nonlinear Problems by the Homotopy Analysis Method," Computational Mechanics, Vol. 20, No. 6, 1997, pp. 530-540. doi:10.1007/s004660050273

[17] S. J. Liao, "Notes on the Homotopy Analysis Method: Some Definitions and Theorems," Communications in Nonlinear Science and Numerical Simulation, Vol. 14,
No. 4, 2009, pp. 983-997. doi:10.1016/j.cnsns.2008.04.013

[18] A. Mehmood, S. Munawar and A. Ali, "Comments to: " Homotopy Analysis Method for Solving the MHD Flow over a Non-Linear Stretching Sheet," Communications in Nonlinear Science and Numerical Simulation, Vol. 14, No. 6, 2009, pp. 2653-2663. doi:10.1016/j.cnsns.2009.12.039 